\title{
Ueber die Verbreiterung der photographischen Sternscheibchen.
}

\author{
Von Prof. $\mathcal{F}$. Sckeiner.
}

In den letzten Jahren sind von verschiedenen Seiten Untersuchungen über die Abhängigkeit des Durchmessers der photographischen Sternscheibchen von Intensität und Expositionsdauer angestellt. worden, die zu verschiedenartigen Formeln geführt haben, welche die Erscheinung innerhalb mehr oder weniger enger Grenzen mit genügender Genauigkeit darstellen. Als Ursache für die Verbreiterung der Sternscheibchen bei wachsender Intensität oder Expositionszeit ist von mir zuerst auf die Reflexion des Lichtes von dem primär beleuchteten Bildpunkte aus in die Schicht hinein aufmerksam gemacht worden, ${ }^{*}$ ) und man scheint diese Erklärung ziemlich allgemein angenommen zu haben. Ich habe weiter versucht, unter Verwendung des einfachen Absorptionsgesetzes die von mir empirisch festgestellte lineare Beziehung zwischen Durchmesser und Grössenclasse theoretisch abzuleiten, und neuerdings hat Herr Harzer eine ähnliche Untersuchung $\dagger$ ) durchgeführt unter Hinzufügung einer Art von sichtleitung*, welche jedoch wesentlich nur von mathematischem Interesse sein dürfte.

Herr Max Wolf hat nun zuerst durch einen sehr interessanten Versuch gezeigt, dass die Lichtreflexion allein nicht zur Erklärung der Verbreiterung ausreicht. Er liess den Bildpunkt eines Sternes auf einen schmalen, undurchsichtigen Gitterstrich fallen, so dass auf der Platte überhaupt kein primärer Lichtpunkt vorhanden war. Trotzdem erschienen auf beiden Seiten des Striches die Segmente des Sternscheibchens und zwar von derselben Grösse wie bei Aufnahmen ohne Gitterstrich. Damit war definitiv bewiesen, dass das Scheibchen nicht durch Reflexion und auch nicht durch sichtleitung * vom primären Punkte aus entstanden war. Ein weiterer Versuch bestand darin, dicht neben dem Brennpunktsbilde des Sterns die photographische Schicht durch ein Stäubchen oder dergl. zu bedecken, wobei dann an dieser Stelle kein Lichteindruck auf der Platte entstand: das Stäubchen hatte einen Schatten geworfen. Hieraus folgt, dass die Verbreiterung von Licht herrührt, welches aus der Richtung vom Objective her kommt. Diese sehr bemerkenswerthen Versuche des Herrn Wolf haben nebenbei ergeben, dass auch eine innere Lichtreflexion stattfindet, wie dies ja auch den Eigenschaften der photographischen Schicht nach sein muss, dass sie aber nur in geringem Maasse zur Verbreiterung beiträgt.

Ich habe nun kürzlich einige Experimente angestellt, welche die- Resultate des Herrn Wolf auf anderem Wege bestätigen und ausserdem geeignet sind, der Sache rechnerisch näher zu treten.

Wenn die Verbreiterung 'der Sternscheibchen von der Beschaffenheit des vom Objective kommenden Lichtes herrührt, so muss sich ein wesentlicher Unterschied zwischen der Wirkung eines durch eine Linse oder einen Spiegel erzeugten primären Lichtpunktes und eines solchen zeigen, bei welchem jeglicher Lichtvorgang vor der empfindlichen Schicht ausgeschlossen ist, also eines durch eine feine Oeffnung erzeugten Lichtpunktes.

Eine feine Oeffnung, welche diese Bedingung erfüllt, also auch eine Diffractionswirkung ausschliesst, erhält man leicht auf folgende Weise. In eine kräftige Messingscheibe bohrt man einen Conus ein, bis dessen Spitze die gegenüber liegende Fläche nahe erreicht, welch' letztere man alsdann bis zur Spitze des Conus vorsichtig abschleift; man kann auf diese Weise beliebig feine kreisrunde Oeffnungen herstellen, deren scharfkantige Begrenzung in der Ebene der Metallscheibe liegt. Drückt man gegen letztere die empfindliche Schicht einer photographischen Platte an und belichtet mit nahe parallelem Lichte durch die conische Oeffnung, so sind wegen der innigen Berührung von Kante und Schicht merkliche Diffractionen ausgeschlossen, und es kann, wenn die Reflexwirkung von der Rückseite der photographischen Platte in der üblichen Weise beseitigt wird, eine Lichtwirkung auf neben dem primär beleuchteten Punkte gelegene Theile der Platte nur noch durch Reflexe innerhalb der Schicht stattfinden.

Mit einer derartigen Vorrichtung angestellte Versuche ergaben nun, dass auch hierbei die Durchmesser der entstehenden Scheibchen mit wachsender Intensität oder Expositionszeit zunehmen, dass also mit Sicherheit eine Verbreiterung durch innere Reflexion stattfindet, dass aber die Scheibchen relativ sehr klein bleiben.

Um die Durchmesser der durch Linsen und durch Oeffnungen erhaltenen Scheibchen mit einander vergleichen zu können, ist es erforderlich, die Lichtintensitäten der beiden primären Scheibchen zu kennen. Im Allgemeinen dürfte die Erlangung dieser Kenntniss aber grosse Schwierigkeiten bereiten, und nur in einem Specialfalle, in welchem die beiden Intensitäten einander gleich werden, ist die Vergleichung ohne Weiteres möglich, nämlich dann, wenn Intensität und Expositionszeit genügend gross sind, um eine beginnende Solarisation des primären Scheibchens zu

$$
\text { *) A. N. } 2884 . \quad \text { †) A. N. } 3104 .
$$


erzeugen; die Mitten der verbreiterten Scheiben werden alsdann wieder hell, sowohl bei Sternen als auch bei den durch Oeffnungen erzeugten Scheiben. Bei einer Reihe von Aufnahmen bei zunehmender Expositionszeit lässt sich der Beginn der Solarisation mit ziemlicher Sicherheit erkennen, und damit ist ein gemeinschaftliches Maass für die vom Lichte geleistete Arbeit gegeben. Wenn man hierbei die Intensitäten noch so wählt, dass die Expositionszeiten bei beiden Methoden nicht zu sehr von einander verschieden sind, so sind auch die wegen der Nichtproportionalität von Zeit und Intensität zu befürchtenden Fehler nicht von merklichem Betrage.

Ich habe nun folgende Durchmesser für die verbreiterten Scheibchen im. Beginne der Solarisation erhalten:

\begin{tabular}{lccc} 
& \multicolumn{2}{c}{ Durchmesser } & Durchm. des \\
in mm & in Bogensec. & prim. Scheibch. \\
Photogr. Refractor & 0.98 & $60^{\prime \prime}$ & $0.05 \mathrm{~mm}$ \\
Voigtländer Euryskop & 0.88 & $480^{\prime \prime}$ & 0.05 \\
Feine Oeffnung & 0.20 & - & 0.06
\end{tabular}

Hiernach ist beim Refractor und beim Euryskop die Solarisation bei 16 - bis 18 maliger Verbreiterung des primären Scheibchens eingetreten, bei den durch Oeffnungen erzeugten Scheibchen schon bei 3-bis 4 maliger, und es kann also nicht mehr dem geringsten $Z$ weifel unterliegen, dass die Lichtreflexion im Innern der Schicht nur einen verhältnissmässig geringen Beitrag zur Verbreiterung der Sternscheibchen liefert.

Hierbei lässt sich gleichzeitig auch genähert feststellen, wie sich die Intensitäten in der Mitte der solarisirten Scheibchen und an dem äussersten Rande, wo nur noch eben eine Lichtwirkung stattgefunden hat, zu einander verhalten. So beginnt z. B. für den Refractor die Solarisation der Mitte (einstündige Expositionszeit) bei Sternen der 3 . bis 4. Grössenclasse, und in derselben Zeit erscheinen als schwächste Sterne, von der Intensität des Randes der verbreiterten Sternscheibchen, die Sterne der 12. bis I3. Grössenclasse; die Helligkeit des Randes ist also um 9 bis ro Classen geringer als die der Mitte, die Intensitäten verhalten sich demnach annähernd wie I $\mathrm{zU} 5000$ bis 10000 . Man ersieht hieraus, dass hier Intensitätsunterschiede in Frage treten, wie solche bei optischen Untersuchungen überhaupt nicht vorkommen; zur Erklärung der Erscheinung der Verbreiterung missen also Factoren in Rechnung gezogen werden, auf welche man sonst bei Fernrohrobjectiven nicht Rücksicht nimmt. Ich werde im Folgenden einen Erklärungsversuch an der Hand der hier für den photographischen Refractor geltenden Zahlen geben.

Von den in Frage tretenden Factoren könnte man in erster Linie an die das Mittelbild umgebenden Diffractionsringe denken. Ich habe schon früher*) gezeigt, dass für das von Gothard'sche Spiegelteleskop die Diffractionsringe nicht die Hauptursache der Verbreiterung sind; dasselbe gilt auch für den photographischen Refractor. Das Intensitätsverhältniss von $1 / 5000$ bis $1 / 10000$ wird beim 7 . bis 9 . Ringe erreicht, die bei dem genannten Instrumente einen Halbmesser von 10 " bis 12 " haben. Ich habe oben dieselbe Intensität für einen Radius der Scheibchen von 30" angegeben, die Diffractionsringe allein könned also nur bis zur Hälfte der verbreiterten Scheiben gewirkt haben.

Man könnte dann weiter an den Einfluss der un. vollkommenen Achromasie denken; dieser Gedanke ist um so mehr berechtigt, als bei Aufnahmen mit für optische Strahlen achromatisirten Objectiven überhaupt keine scharfen Sternscheibchen entstehen, und hier zweifellos die sehr grossen blauen und violetten Abweichungskreise hauptsächlich die Verbreiterung bewirken. Bei dem Potsdamer photographischen Objective sind jedoch die Strahlen von $F$ bis ins äusserste Ultraviolett (das Gebiet der photographisch wirksamen Strahlen) sehr gut vereinigt, und zwar derart, dass der Halbmesser des stälksten Abweichungs. kreises $-F-$ nur etwa $0.1 \mathrm{~mm}=6$ " beträgt. Erst bei $C$ erreicht dieser Halbmesser den Betrag von $0.5 \mathrm{~mm}=30^{\prime \prime}$, wie sich leicht durch Anwendung rothempfindlicher Platten constatiren lässt.

Der Einfluss der sphärischen Aberration ist bei dem besprochenen Objective wie bei allen guten astronomischen Objectiven sehr gering und beträgt für die Randstrahlen höchstens $0.05 \mathrm{~mm}=3$ ".

Bei allen nicht verkitteten Objectiven, bei denen die Krümmungsradien der beiden inneren Flächen nicht sehr von einander verschieden sind, entsteht durch doppeite Reflexion in der Nähe des Focalbildes ein zweites Bild, dessen Distanz vom Focalbilde von der Differenz der beiden Krümmungshalbmesser abhängt. Bei Bildern ausserhalb der optischen Axe liegt dieses Reflexbild zwar nicht genau centrisch zum eigentlichen Bilde, man könnte jedoch diesem, in der wahren Brennfläche als Scheibchen erscheinenden Bilde die Ursache der Verbreiterung zuschreiben. Es lässt sich aber leicht zeigen, dass dies nicht der Fall ist. Die Intensität des zweiten Bildes ist wegen seiner Entstehung durch doppelte Reflexion zu annähernd $1 / 400$ anzunehmen. Nimmt man den Halbmesser des Bildes selbst zu I"!5 an, ein jedenfalls eher zu grosser als zu kleiner Werth, so reducirt sich seine Flächenintensität aber auf etwa $1 / 160000$. wenn es eine solche Distanz vom Focus hat, dass es in der Brennfläche als Scheibchen mit dem geforderten Halbmesser von 30" erscheint.

Ich glaube, hiermit gezeigt zu haben, dass keine der bei einem Objective rechnungsmässig zu verfolgenden Fehlerquellen für sich allein eine Erklärung für die Verbreiterung. der photographischen Sternscheibchen geben kann, und dass auch ihr Gesammteinfluss nicht die bei langen Expositionszeiten und grossen Intensitäten auftretende starke Verbreiterung zu erklären vermag. $\mathrm{Da}$ ausserdem nach Angabe des Herrn Dr. Steinheil die Flächen des ungefassten Objectivs. nicht um Beträge von $1 / 100000 \mathrm{~mm}$ von der wahren Kugelgestalt abweichen, so können zur Erklärung der Verbreiterung nur noch die unregelmässigen Fehler des Objectivs herbeigezogen werden. Hierbei hat man zwei Arten derselben zu unterscheiden, einmal die Rauhigkeiten der Oberflächen, kleine Schlieren, Luftbläschen etc., welche dem 
Objective unveränderlich angehören, und in zweiter Linie Deformationen des Objectivs durch die Fassung desselben. Was die ersteren angeht, so ist es klar, dass sie bewirken, dass ein Theil des auf das Objectiv fallenden Lichtes nicht im Bildpunkte vereinigt wird, sondern als zerstreutes Licht sich ïber das ganze Gesichtsfeld verbreitet; man erkennt diese Erscheinung sehr deutlich, wenn man schräg auf ein von der Sonne beschienenes Objectiv blickt.

Da aber kleinere Fehler naturgemäss häufiger vor* kommen als grössere, so sind geringere Abweichungen der Strahlen vom regelmässigen Gange häufiger als grosse, und folglich ist die Intensität des zerstreuten Lichtes in der Nähe des Bildpunktes grösser als weiter von demselben. Diese Ursache kann augenscheinlich eine unbegrenzte Ausbreitung der Sternscheibchen in Gefolge haben.

Die Fassung des Objectivs äussert sich im Strahlengange folgendermaassen. Alle mir bekannten Objective (auch Spiegel), welche an drei Punkten gefasst sind, oder bei welchen die unmittelbare Berührung der beiden Linsen durch drei Stanniolstreifen verhindert ist, liefern photographische Scheibchen, von welchen sechs Strahlen ausgehen, die nach den drei Druckpunkten orientirt sind. Es ist also anzunehmen, dass bei solchen Objectiven durch die Vertheilung des Druckes auf drei Punkte Einsattelungen entstehen, welche bewirken, dass in den Richtungen auf die Druckpunkte zu die Vereinigung der Strahlen theilweise in grösserer Entfernung vom Bildpunkte erfolgt. Der Umstand, dass nicht drei Strahlen, sondern sechs entstehen, dass also jeder Strahl eine Fortsetzung über den Bildpunkt hinaus erfährt, und besonders, dass diese Fortsetzung fast genau gleich dem eigentlichen Strahl in Bezug auf Länge und Intensität wird, scheint darauf hinzudeuten, dass auch eine Diffractionswirkung hierbei mitspielt; dass letztere allein die Ursache der Strahlen bilden sollte, ist für das Objectiv des photographischen Refractors völlig ausgeschlossen, da hier die Stanniolblättchen nicht in die freie Oeffnung heraustreten. Objective, die voll gefasst sind, z. B. das Voigtländer'sche Euryskop, zeigen die sechs Strahlen natürlich nicht; es ist aber anzunehmen, dass der allseitige Druck auf den Rand auch eine allgemeine Deformation des Objectivs bewirkt, ähnlich wie wenn die Flächen des ungefassten Objectivs Abweichungen von der regelmässigen Kugelgestalt besässen. Es mag dies theilweise die Ursache für die im Winkelwerth 8-mal stärkere Ausbreitung der Sternscheibchen im Euryskop gegenüber dem Refractor sein, zum anderen Theile sind hierfür gewiss die etwas weniger exacte Ausfuhrung der Flächen und die Zusammensetzung aus vier Linsen maassgebend.

Auch bei der Fassung durch drei Druckpunkte muss eine geringe Deformation des gauzen Randes erfolged und somit ein Beitrag zur allgemeinen Verbreiterung der Sternscheibchen geliefert werden. Um dies experimentell zu prüfen, habe ich beim photographischen Refractor eine Central- und eine Randblende, welche gleiche Flächenräume abblendeten, benutzt.

Die Randblende bewirkte nun zunächst eine Verkleinerung des primären Scheibchens, entsprechend der theilweisen Aufhebung der sphärischen Aberration, gleichzeitig auch eine im Verhältniss nur sehr geringe Abnahme der photographischen I.ichtstärke, weil die durch die Randscheibe des Objectivs gehenden Strahlen wesentlich nur eine Verbreiterung des primären Bildes bewirken und nur eine geringe Verstärkung der Flächenintensität desselben; ich werde auf diese Erscheinung noch zurückkommen. Die an sich nur geringe Veränderung des primären Scheibchens kann auf das Verhalten des verbreiterten Scheibchens nur einen geringen oder ganz verschwindenden Einfluss ausüben, trotzdem zeigten die Versuche, dass bei gleichen Expositionszeiten die verbreiterten Scheibchen mit Randblende im Verhältniss von $3: 2$ grösser wurden als bei Centraiblende. Diese Erscheinung lehrt also, dass die Randpartien des Objectivs mehr zur Verbreiterung beitragen als die mittleren Theile, dass dies aber, da die Flächen des ungefassten Objectivs am Rande gerade so gut sind wie in der Mitte, eine Folge der durch die Fassung bedingten Deformation des Objectivs ist.

Die verbreiterten Scheibchen besitzen eine Eigenschaft, auf welche bisher $\mathrm{m}$. E. zu wenig Gewicht gelegt worden ist. Sie sind nämlich zuerst sehr scharf begrenzt, und erst von einem gewissen Durchmesser an beginnt der Rand verwaschen $z u$ werden, bis schliesslich bei sehr grossen Scheiben die Verwaschenheit eine grössere Ausdehnung besitzt als der schwarze Kern. Unter der nicht zu bezweifelnden Annahme, dass bei einer gegebenen Intensität eine untere Grenze der Expositionszeit existirt, unterhalb welcher keine in die Erscheinung tretende Wirkung auf die empfindliche Schicht ausgeubt wird, oder ungekehrt, bei einer gegebenen Expositionszeit eine entsprechende untere Grenze der Intensität, ist nur die folgende Erklärung für die obige Eigenthümiichkeit der Scheibchen zulässig: Der Intensitätsabfall in der Nähe des Bildpunktes ist ein so steiler, dass einer sehr geringen Aenderung der Entfernung eine so starke Aenderung der Intensität entspricht, dass der Uebergang vom völlig aus. exponirten Bromsilber bis zum unzersetzten innerhalb einer sehr kleinen Strecke erfolgt, während der Intensitătsabfall in grösserer Entfernung vom Bildpunkte immer flacher wird. Die Intensitätscurve hat also die Form der in der beistehenden schematischen Figur punktirt gezeichneten steilen Curve, bei welcher die Ordinatenaxe in den Rand des primären Sternscheibchens verlegt ist.

Ich denke mir

nun die Entstehung

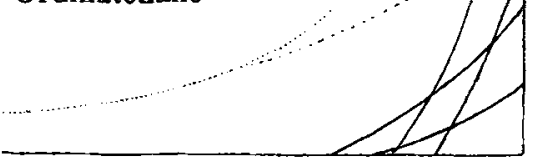

dieser Curve folgendermaassen: In der Nähe des Bildpunktes wirken auf die Verbreiterung zunächst die ersterwähnten Ursachen, also Diffraction, sphärische und chromatische Aberration und innere Reflexion in der Schicht; diese Intensitätscurven mögen in der Figur durch die ausgezogenen Linien angedeutet sein; sie erreichen die Wirkungsschwelle schon alle nahe beim Bildpunkte. Die gestrichelte Curve möge nun den Intensitätsverlauf der 
durch die unregelmässigen Fehler des Objectivs und die Fassung verursachten Lichtzerstreuung darstellen; diese Curve hat die Eigenschaft, erst in weit grösserer Entfernung von der Axe den Schwellenwerth zu erreichen, also sehr viel flacher zu verlaufen. Die Gesammtwirkung der fünf Curven giebt die oben festgestellte Intensitätscurve der verbreiterten Scheibchen.

Es ist nun auch ohne Weiteres einleuchtend, dass für jedes Objectiv je, nach seiner Construction, der Feinheit seiner Bearbeitung und der Art seiner Fassung die Curve anders ausfallen wird, und hieraus sind die Verschiedenheiten in den Formeln für die Verbreiterung der Scheibchen als Function von Zeit und Intensität zu erklären, welche verschiedene Beobachter bisher erhalten haben Ebenso ergiebt sich unnittelbar die Richtigkeit meiner schon früher ausgesprochenen Behauptung, dass keine der bisherigen hierfür gefundenen Formeln eine physikalische Bedeutung hat, sondern dass sie nur als Interpolationsformeln $\mathrm{zu}$ betrachten sind, dass also das praktischste Verfahren bei photographisch-photometrischen Untersuchungen stets die graphische Ausgleichung sein wird. Diese Bemerkung bezieht sich auch auf diejenigen Formeln, welche gewisse Instrumentenconstanten enthalten, z. B. die Objectivöffnung, und welche den meisten Anschein von physikalischer Bedeutung besitzen.

Ich möchte noch einige Bemerkungen über die Lichtstärke photographischer Objective anschliessen. Für die Lichtstärke bei Flächenabbildungen (Nebelflecken) ist wesentlich nur das möglichst kleine Verhältniss von Brennweite zu Oeffnung maassgebend, bei Abbildung von Punkten (Fixsternen) dagegen die möglichst grosse absolute Oeffnung. Während diese bei optischen Beobachtungen die allein maassgebende ist, kommt bei photographischen Objectiven die Bedingung der möglichst guten Vereinigung der Strahlen hinzu. Ein Objectiv, welches das aufgefallene Licht in einem primären Scheibchen von I" Durchmesser vereinigt, hat photographisch ungefähr die vierfache Lichtstärke gegenüber einem sonst gleichen, welches aber Scheibchen von 2" erzeugt. Von Objectiven gleicher Oeffnung, aber verschiedener Brennweite ist dasjenige am lichtstärksten, welches die Strahlen in einem linear gemessen kleinsten Scheibchen vereinigt.

Da, wie oben gezeigt, die Randabblendung eine Verkleinerung des primären Scheibchens bewirkt, welche für die Feinheit der Ausmessung wesentlich ist, und gleichzeitig die Lichtstärke nur wenig abnimmt - selbst für das vorzügliche Steinheil'sche Objectiv tritt bei der Reduction der Oeffnung auf $2 / 3$ nur ein Verlust von 0.1 bis 0.2 Grössenclassen ein -, so ist es für viele Beobachtungen vortheilhaft, das Objectiv abzublenden, wobei durch Versuche festzustellen ist, bei welcher Oeffnung die kleinsten primären Scheibchen entstehen. Gleichzeitig wird hierdurch auch die Verbreiterung geringer, was ebenfalls für die Messung günstig ist.

Es würde wohl eine Aufgabe für die Optiker sein, für grössere Objective Fassungen herzustellen, bei welchen die nöthige Sicherheit der Befestigung mit einem Minimum von Druck erreicht ist, und es würde hierbei vielleicht in Erwägung zu ziehen sein, ob man nicht gleich die $\mathrm{Ob}$, jećtive so construiren will, als ob aus einem grösseren die Mitte herausgeschnitten worden wäre; sie also relativ dick herzustellen behufs besseren Widerstandes gegen den deformirenden Druck der Fassung. Es ist immerhin denkbar, dass die Nachtheile, welche die grössere Glasdicke mit sich bringt, durch die erwähnten Vortheile überwogen werden. Jedenfalls erscheint es mir nicht fraglich, dass bei den heutigen Fassungen die grösseren Objective auch nicht an; nähernd mehr die Güte der Flächen nach dem Rande zu besitzen, wie sie der Optiker mit grossem Aufwand von Mühe hergestellt hat.

Potsdam, Kgl. Observatorium, 1893 April.

F. Scheiner.

\section{Bemerkung zu Herrn Kobold's Aufsatz}

„Ueber die Bestimmung der eigenen Bewegung des Sonnensystems" in A. N. 3163-64.

\section{Von Paul Harzer.}

Das in Herrn Kobold's Aufsatz in A. N. $3^{16} 3^{-6} 4_{4}$ vorkommende Problem: zu $n-x$ Punkten, die auf der Kugeloberfläche vom Radius I beliebig gegeben sind, einen $n^{\text {ten }}$ Punkt derart zu bestimmen, dass die Summe der Cosinus quadrate der Winkel zwischen je einem der $n$ - I Punkte und dem zu bestimmenden $n^{\text {ten }}$ Punkte ein Max. oder Min. werde, ist, anstatt mit den zwei sphärischen Coordinaten, wohl einfacher, jedenfalls aber eleganter, mit den drei rechtwinkligen Coordinaten zu lösen. An Bekanntes erinnernd, führe ich die folgende Lösung des Problems an:

Es seien $x_{1 \alpha}, x_{2 \alpha}, x_{3 \alpha}$ die rechtwinkligen Coordinaten der Punkte, indem sich die Werthe $\alpha=1,2 \ldots n-1$ auf die gegebenen, $\alpha=n$ auf den gesuchten Punkt beziehen.
Es muss dann der Ausdruck:

$$
2 f=\sum_{\alpha=1}^{\alpha=n-1}\left(x_{1 \alpha} x_{1 n}+x_{2 \alpha} x_{2 n}+x_{3 \alpha} x_{3 n}\right)^{2}
$$

mit Rücksicht auf die Bedingung

$$
2 \varphi=x_{1 \dot{n}}{ }^{2}+x_{2 n}{ }^{2}+x_{3 n}{ }^{2}-1=0
$$

zu einem Max. oder Min. gemacht werden. Die Anwendung der allgemeinen Vorschriften der Variationsrechnung ergiebt die folgende Regel zur Lösung des Problems:

Es sei : 